\title{
Comparison of Ion-Specimen Interactions in Silicon \& Molecular Materials
}

\author{
D.J. Stokes ${ }^{*} \&$ A.L. Bleloch ${ }^{* *}$ \\ *Biological \& Soft Systems, Cavendish Laboratory, University of Cambridge, Dept. of Physics, JJ \\ Thomson Avenue, Cambridge, CB3 0HE, UK \\ ${ }^{* *}$ SuperSTEM Laboratory, CLRC Daresbury, WA4 4AD, UK
}

Focused ion beam (FIB) is a well-known technique for semiconductor device edit and mask repair, and advanced lithography methods are being developed that utilise focused ions for nano-fabrication of complementary metal-oxide-semiconductors and functional quantum devices [1]. Currently of interest is nano-scale implantation of 'low energy' focused $\mathrm{Ga}^{+}$ions $\left(E_{0} \leq 30 \mathrm{keV}\right)$ in resist materials such as PMMA, and their advantageous role in subsequent processing chemistry.

FIB techniques combined with electron microscopy are increasingly being used for characterisation of polymers and life sciences specimens. Site-specific cross-sections as well as serial sectioning and imaging are providing new approaches to SEM studies of these materials [2], giving access to internal microstructure and enabling three-dimensional visualisation of bulk specimens.

FIB-prepared thin specimens offer a means for TEM and STEM analysis at the nanoscale, and there are many examples relating to semi-conducting materials. Here, the interactions of ions with crystalline materials are a concern, as they tend to introduce amorphous surface layers, up to tens of nanometers in thickness, depending on milling conditions. With the requirement for specimen thicknesses of $30-100 \mathrm{~nm}$ for high-resolution imaging and chemical analysis (i.e. HRTEM/STEM + EELS), ways to limit this damage have necessarily been identified. For example, a recent HRTEM study of side-wall damage in silicon has shown that the amorphous layer can be reduced to as little as $0.5-1.5 \mathrm{~nm}$ if the specimen is FIB-polished with $\mathrm{Ga}^{+}$at $E_{0}=2 \mathrm{keV}$ [3].

The extent and nature of ion implantation and any damage in molecular materials such as polymers and biological specimens is not so well documented, and hence there is a need to elucidate the relevant mechanisms. We can make some estimates using Monte Carlo simulation codes such as TRIM and SRIM 2003 [4], complemented by experimental evidence. Calculation of the mean projected longitudinal range $\mathrm{R}_{\mathrm{p}}$ for $\mathrm{Ga}^{+}$ions at an angle of incidence $\theta=0^{\circ}$ (normal to the specimen surface) is shown in Fig. 1(a) for silicon, bone, pancreas and PMMA. The data shown takes into account differences in binding energies for compounds compared to elemental solids, to help improve accuracy when approximating the ion range in molecular solids. HAADF STEM has been used to show that normally incident $30 \mathrm{keV} \mathrm{Ga}{ }^{+}$ions are implanted to a depth of 50nm in PMMA resist [1], consistent with these calculations.

At high angles of incidence, i.e. $\theta \sim 90^{\circ}$ (almost parallel to the milled surface of interest), the longitudinal range of $\mathrm{Ga}^{+}$ions becomes quite similar for these materials. Simulations yield implantation on the order of $\sim 1 \mathrm{~nm}$ for $E_{0}=1 \mathrm{keV}$ to $\sim 10 \mathrm{~nm}$ for $E_{0}=30 \mathrm{keV}$. Since experimental data shows that $30 \mathrm{keV} \mathrm{Ga}{ }^{+}$ions result in roughly $20 \mathrm{~nm}$ of amorphisation in silicon [3], the difference between theory and experiment suggests that we must look for effects in addition to ion implantation. 
In fact we must include the recoil motion of atoms or molecules of the target material, which in turn creates damage. Calculation of the entire collision cascade as a function of $E_{0}$ for $\theta \sim 90^{\circ}$ (Fig. 1(b)) suggests that the total damage layer for $30 \mathrm{keV} \mathrm{Ga}^{+}$ions in silicon should indeed be roughly $20 \mathrm{~nm}$. Fig. 2 shows schematically both implanted $\mathrm{Ga}^{+}$and displaced molecules (PMMA) and atoms (Si). The similarity in implantation depths for $\theta=90^{\circ}$ is easily seen from Fig. 2, and it becomes clear that the displacement of recoiled species accounts for the difference in total damage between these materials.

Whilst milling at low beam energy significantly reduces damage, there are other factors that require investigation for optimised milling of molecular materials. Further work includes consideration of, for example, self-annealing due to polymer chain movement, the influence of reactive gases on surface binding energies, and quantification of tertiary damage due to inelastic electronic processes in the bulk. Experimental verification is challenging and requires a broad range of techniques. Initial data will be presented, using EELS to assess $\mathrm{Ga}^{+}$implantation and structural rearrangement.

\section{References}

[1] Arshak. K. et al, Microelectronic Engineering, 78-79 (2005) 39-46

[2] Stokes, D.J., Morrissey, F. \& Lich, B.L., Inst. Phys. Conf. Ser. (2006) (in press)

[3] Giannuzzi, L.A., Geurts, R. \& Ringnalda, J., Microsc. Microanal. 11 (Suppl 2) (2005) 828-829

[4] Ziegler, J.F. and Biersack, J.P., SRIM 2003 (see www.srim.org)

[5] DJS gratefully acknowledges the financial support of FEI Company

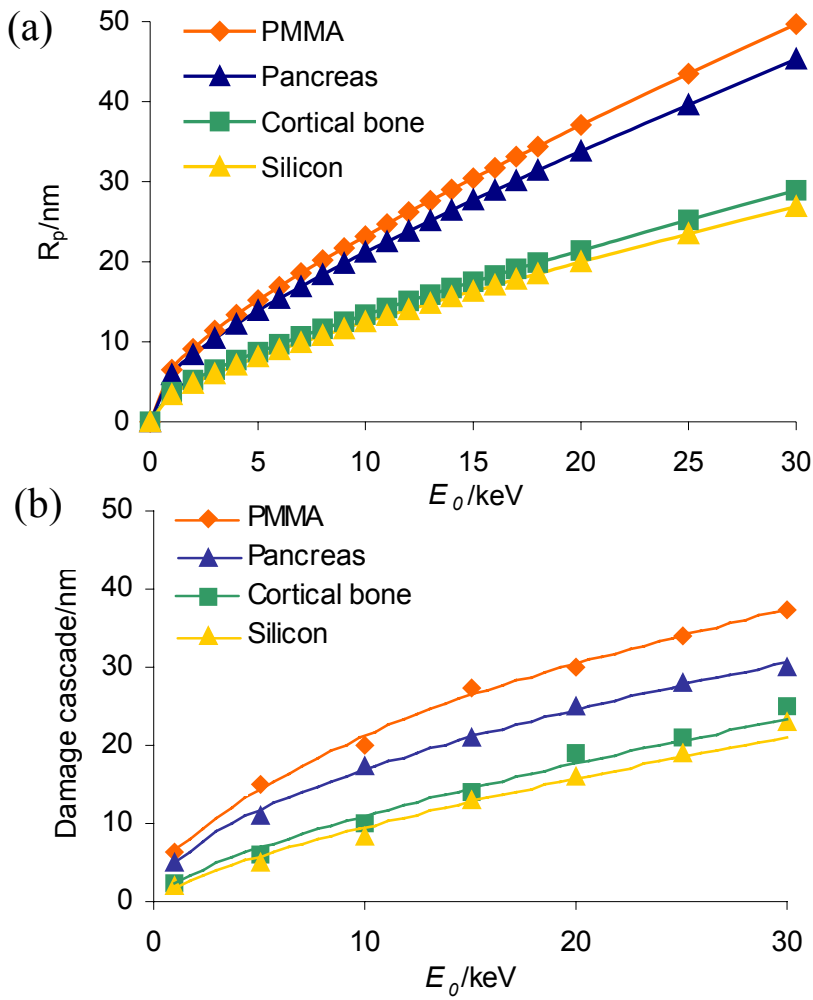

Fig. 1. (a) Calculated ion implantation for $\theta=0^{\circ}$

(b) Calculated total damage for $\theta \sim 90^{\circ}$

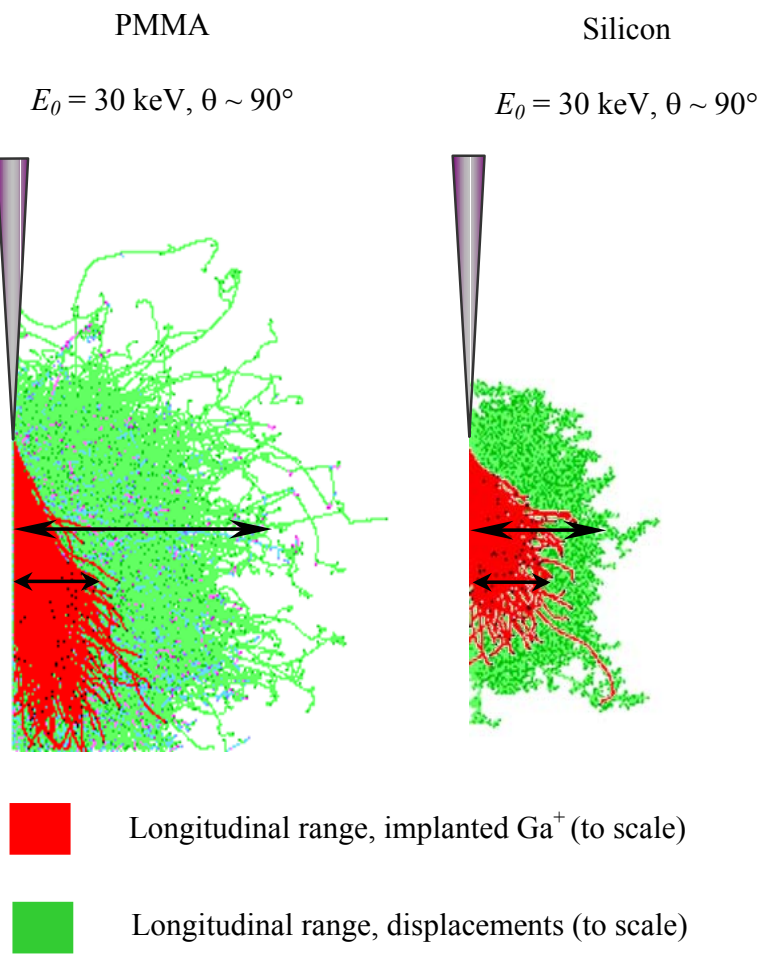

Fig. 2. Models of implantation and displacement 